\title{
Synthetic Spectra for Carbon-Rich Long-Period Variables
}

\section{RITA LOIDL ${ }^{1}$, BERNHARD ARINGER ${ }^{1}$, UFFE JøRGENSEN ${ }^{2}$, SUSANNE HÖFNER ${ }^{1}$ and JOSEF HRON ${ }^{1}$}

${ }^{1}$ Institut für Astronomie der Universität Wien, Vienna, Austria

2 Niels Bohr Institute, Copenhagen, Denmark

We have computed exploratory synthetic spectra for carbon-rich longperiod variables by using the dynamical model atmospheres of Höfner et al. (see their abstract in this volume) as input for the spectral synthesis code of Jørgensen et al. (e.g. $A \mathscr{E} A, 261,263,1992)$. We compare the atmospheric structure of an extended, hydrostatic model atmosphere computed with the MARCS code with a corresponding hydrostatic initial model of Höfner and a few selected dynamical models. We find an overall agreement between the models in the range where the spectra are formed. Synthetic opacity-sampling spectra of two different models are shown for the three molecules $\mathrm{C}_{2}, \mathrm{CN}$ and $\mathrm{HCN}$ for different phases of the light curve.

Since the symposium, we have compared the synthetic spectra presented here with ISO SWS spectra of the semi-regular carbon star R Scl (Hron et al. 1998, $A \& A, 335, \mathrm{~L} 69$ ) and the carbon Mira T Dra (Loidl et al. 1997, Astrophys. Space Sci., 251, 243).

This work is supported by the Fonds zur Förderung der wissenschaftlichen Forschung under project numbers S7308-AST and S7305-AST. 